\title{
Dynamical evolution of the ICM
}

\section{Giuseppe Tormen}

Dipartimento di Astronomia, Università di Padova, Italy email: tormen@pd.astro.it

\begin{abstract}
We use numerical simulations of galaxy clusters to study the dynamical and thermal evolution of the ICM from high redshift to the present time. We measure the properties of the satellites accreting on the cluster main progenitor and then measure their self-bound mass fraction as a function of time after the merging. We also calculate the mean properties of their orbits and investigate the time evolution of their internal velocity dispersion and gas temperature. We measure and model the mean pericentric and apocentric times, distances and velocities. Finally we show how the properties of the ICM at redshift zero can be understood by decomposing them as a function of the cluster merging history.
\end{abstract}

\section{Properties of satellites at merging time}

Tormen (1997) made an analysis of the properties of dark matter satellites measured at the time $t_{m e r}$ when they first are accreted onto the main progenitor of a galaxy cluster. The main results of this research are the following:

- the infall of satellites onto the main cluster progenitor is very anisotropic. As shown on the left panel of Figure 1, the mean pericenter - measured from the orbits at merging time - is of order 30 percent of the virial radius of the cluster at that time. Orbits are neither too circular, nor very elongated: their circularity $\epsilon=J / J_{c}$ (ratio of the satellite's angular momentum to the angular momentum of a circular orbit with the same energy; $\epsilon=0$ for purely radial orbits, $\epsilon=1$ for purely circular ones) is peaked around $\epsilon=0.5$, and roughly symmetric.

- Massive satellites tend to approach the main progenitor along slightly more radial orbits compared to less massive ones. This is consistent with the fact that most of the mass accreted onto a cluster comes along large scale filaments, where massive satellites are more likely to live.

- The infall pattern of satellites is largely responsible for the final shape and orientation of the cluster mass and velocity distribution, as shown on the right panels of Figure 1. This means that, if we model the shape of the infall pattern with an ellipsoid, this tends to be aligned (typically within 30 degrees) with the mass and velocity ellipsoids.

\section{Properties of merged satellites}

The results presented in this section are throughly discussed in Tormen, Moscardini and Yoshida (2004), where the reader is addressed to for full details of the simulation data and method. After satellites accrete onto the main cluster progenitor, their properties evolve quite rapidly, by the different interaction with the gravitational field and the ICM of the host. Simulations (e.g. Tormen, Diaferio and Syer 1998; Tormen, Moscardini and Yoshida 2004) show that massive sub haloes penetrate deeper into the main cluster, and unbind their dark matter more quickly than less massive ones. The ICM is stripped immediately from small satellites, while the gravitational potential well of massive ones is deep enough to prevent stripping of the hot gas until the whole satellite is destroyed. 

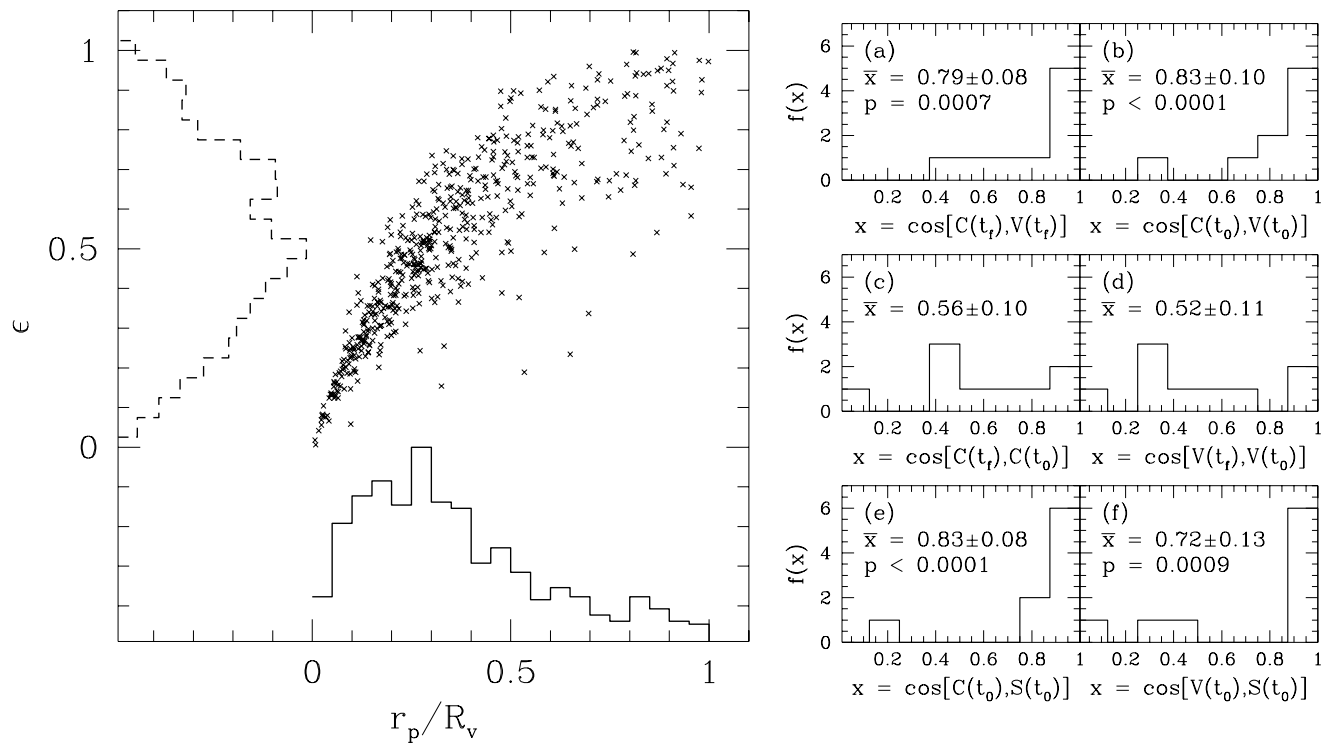
$\mathrm{x}=\cos \left[\mathrm{C}\left(\mathrm{t}_{\mathrm{f}}\right), \mathrm{V}\left(\mathrm{t}_{\mathrm{f}}\right)\right] \mathrm{x}=\cos \left[\mathrm{C}\left(\mathrm{t}_{0}\right), \mathrm{V}\left(\mathrm{t}_{0}\right)\right]$

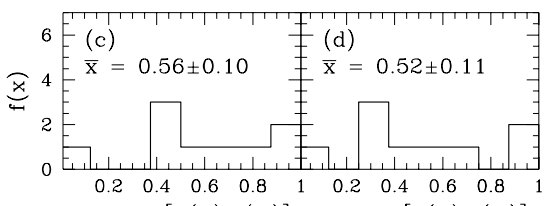
$\mathrm{x}=\cos \left[\mathrm{C}\left(\mathrm{t}_{\mathrm{f}}\right), \mathrm{C}\left(\mathrm{t}_{0}\right)\right] \mathrm{x}=\cos \left[\mathrm{V}\left(\mathrm{t}_{\mathrm{f}}\right), \mathrm{V}\left(\mathrm{t}_{0}\right)\right]$

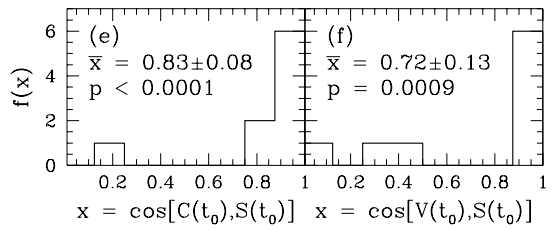

Figure 1. Left: Scatter plot of the orbital circularity $\epsilon$ versus the pericenter $r_{p} / R_{v}$ in units of the virial radius of the main halo progenitor at merging time. The histograms show the projected distributions. Right: Correlation between the major axis of the cluster mass ellipsoid (C), the velocity ellipsoid (V) and the infall pattern of satellites (S). The histograms show distributions of the cosine of misalignment angles between the different ellipsoids. Where relevant, $p$ gives the probability that such a correlation arises by chance, measured by a KS test (from Tormen 1997, reprinted with permission).

In the host halo, thermalization of the dark matter and hot gas accreted through the satellites happens on different timescales for the two components. The left panels of Figure 2 show that the ICM temperature of the particles initially belonging to the satellites increases faster during the 2 Gyr immediately following the merging, and stabilizes or increases more slowly thereafter. Globally the new thermal equilibrium is approached in roughly $2 \mathrm{Gyr}$, almost regardless of the satellite mass (solid bands). On the other hand, the internal velocity dispersion of the satellite dark matter takes longer to increase, and it does so more quickly for massive satellites (hatched bands).

The evolution of the same quantities, for particles which remain bound to the substructure, is quite different, as shown in the right panel of Figure 2. We remind that these particles constitute the cores of satellites, and so are those which would be actually identified as substructure in a simulation or in an observation. The figure shows that the dark matter velocity dispersion of the satellite cores never increases, but actually decreases: satellite cores become colder, the effect being more dramatic for the most massive satellites (bottom right panel). The ICM temperature displays a similar behavior, except for the smallest satellites (upper left), where the potential well is too shallow to shield the satellite ICM from the global heating. This results suggests that one should place particular attention when trying to estimate the mass of substructure from the temperature of its diffuse gas or from the velocity dispersion of its galaxies.

A summary of the orbital properties of satellites at their first pericenter and at the following apocenter are shown in Figure 3. The upper panels show the time in Gyr needed for a satellite to reach its first pericenter after crossing the virial radius of the main cluster (left), the cluster-centric distance at pericenter in units of the cluster virial 

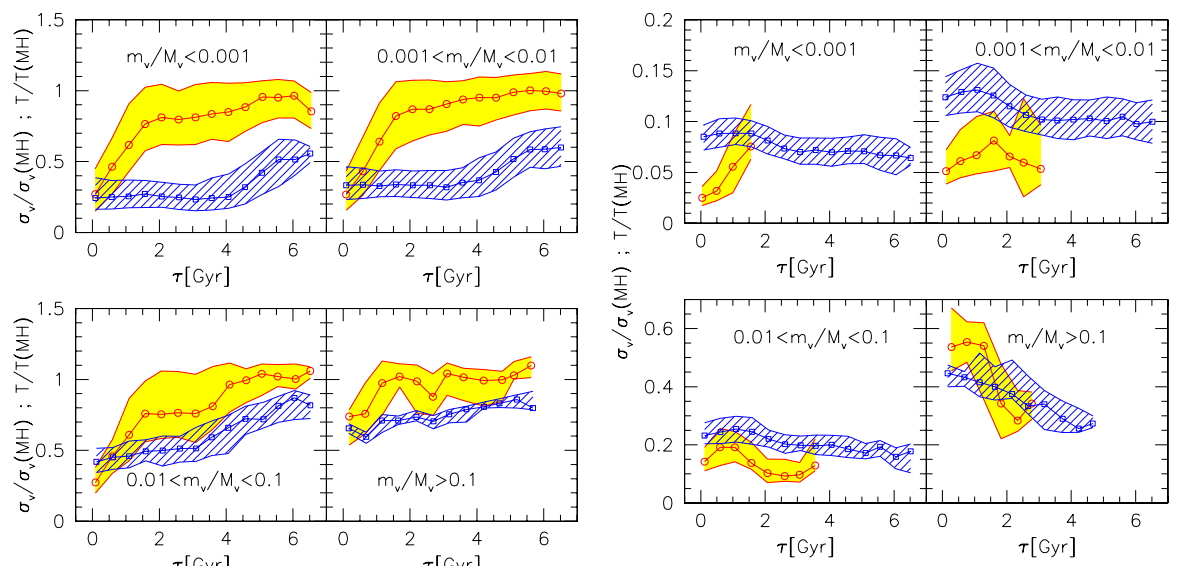

Figure 2. Time evolution of the dark matter velocity dispersion (hatched bands) and of the gas temperature (solid bands). The left panel shows evolution for all particles initially belonging to satellites, while the right panel shows evolution of the particles which at each time $\tau$ are still bound to the satellite. Different sub-panels refer to satellites of different mass, in units of the mass $M_{v}$ of the host cluster measured at merging time. Satellite temperatures and velocity dispersions are normalized by the corresponding value measured for the main cluster at each time $\tau$ after merging (from Tormen, Moscardini \& Yoshida 2004, reprinted with permission).
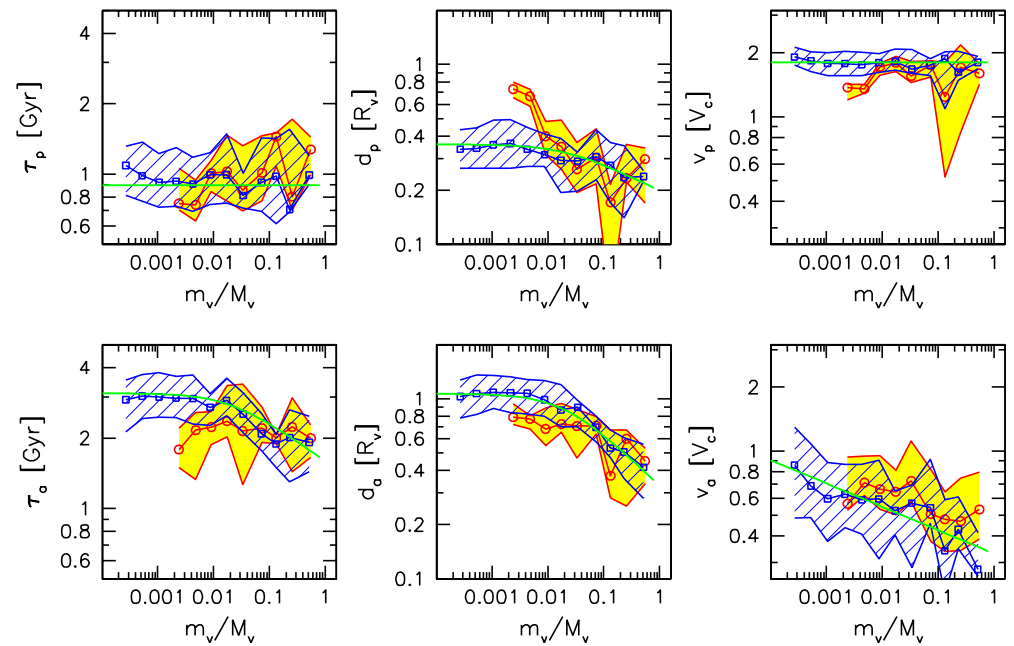

Figure 3. Orbital quantities at first pericentric passage (upper panels) and at the following apocenter (lower panels), shown as a function of the satellite mass with respect to the mass of the main cluster progenitor, $m_{v} / M_{v}$. Solid bands refer to gas particles, hatched bands refer to dark matter. Times are expressed in Gyr, distances are in units of the virial radius of the main cluster at that time, while velocities are in units of the circular velocity of the main cluster at the same time (from Tormen, Moscardini and Yoshida 2004, reprinted with permission).

radius (center) and the corresponding orbital velocity in units of the circular velocity of the main halo (right). In each panel, these quantities are plotted versus the satellite mass in units of the mass of the main cluster. The lower panels show the same quantities at first apocenter. 

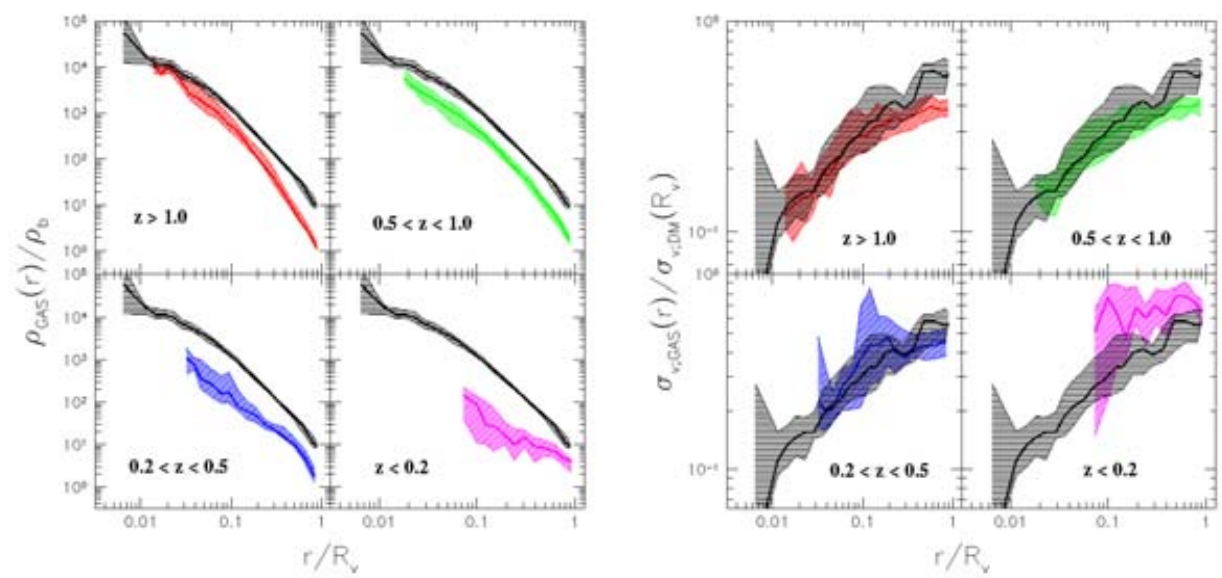

Figure 4. ICM decomposition in redshift: gas density (left plot) and gas velocity dispersion (right plot). In each plot the hatched (black) bands show global mean profiles over our SPH cluster sample (plus or minus one sigma of the mean), which are the same for all four panels in each plot. Colored bands show the mean profile over the cluster sample, but considering only the indicated subset of particles. For example, the upper left panels in each plot contrasts the global profiles with those of particles accreted at redshift larger than one.

We can see that pericentric times are reached in roughly 1 Gyr, regardless of the satellite mass; pericentric distances are smaller for massive satellites, and in rough agreement with those predicted by the initial orbits (Figure 1). Pericentric velocities are also mass-independent, with values around twice the circular velocity of the main halo. On the other hand, massive satellites reach their apocenter more quickly (lower left); they stay closer to the cluster center (lower center), and are significantly slower than small satellites (lower right). These results are consistent with the expectation that massive satellites slow down their motion quickly after their first encounter with the cluster, and merge with the cluster itself after at most a couple of pericentric passages.

\section{Decomposition of the final ICM}

A point of view orthogonal to that taken in the previous section is that of decomposing the ICM of the cluster at redshift zero in subsamples, by selecting the particles that were accreted by the main cluster progenitor at different redshifts. Yet another alternative is that of separating the ICM particles depending on mass of the satellite that carried them at merging time.

A simple study then is to analyze the ICM radial profiles: here we looked at the gas density, temperature, velocity dispersion and entropy. The interest of this experiment is then to be able to link the profiles to the merging history of the cluster, understanding the possible differences in the light of the different dynamical and thermal histories of the different particle subsets.

\subsection{Decomposition in redshift}

Figure 4 shows the mean gas density (left) and velocity dispersion (right) profiles averaged over our cluster sample at redshift $z=0$. The black band is the mean profile $( \pm 1 \sigma$ of the mean) obtained using all the gas particles of each cluster. The colored profiles correspond instead to subsets of particles selected according to the redshift when they 

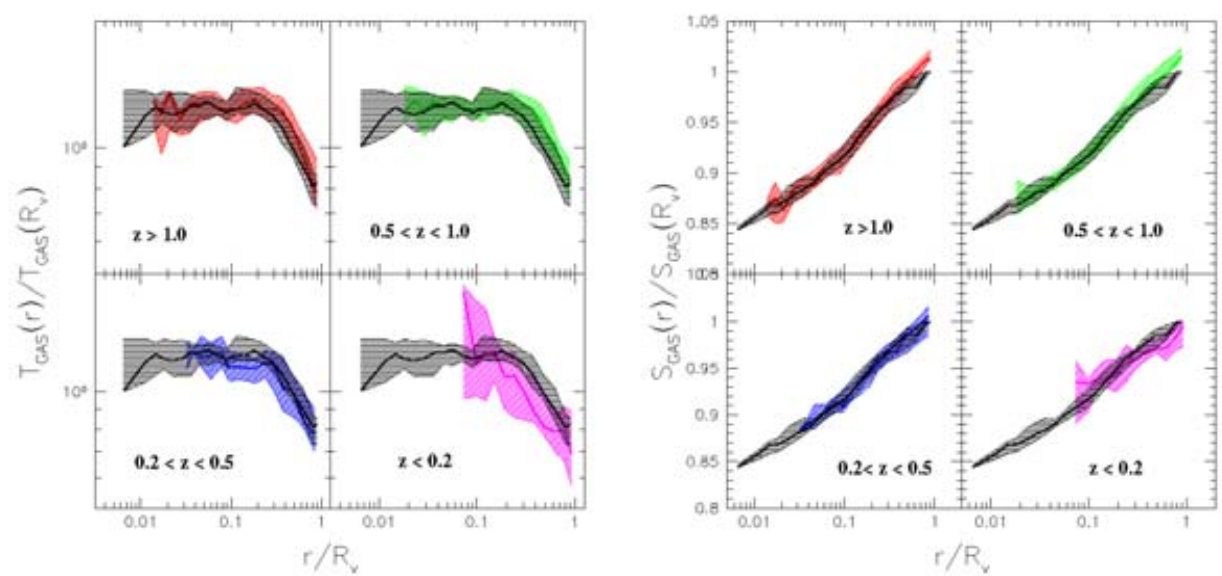

Figure 5. ICM decomposition in redshift: gas temperature (left plot) and entropy (right plot). Here also the hatched black bands correspond to global mean profiles, and the colored ones to profiles made using a subset of particles.

first were accreted by the main cluster progenitor. We immediately see a segregation in mass in the density plot: while particles accreted at redshift $z>1$ (upper left panel) end up closer to the center in the final cluster, and in fact account for most of the gas mass in the inner regions, moving to lower and lower accretion redshifts the gas distribution becomes shallower and shallower, while more and more gas is confined to the external regions of the clusters. In particular, the lower right panel of the left plot indicates that most of the gas found around the virial radius at redshift $z=0$ has been accreted very recently, at $z<0.2$. This trend is effectively a secondary infall signature, as gas accreted at later times is, on average, stopped at larger and larger radii by the outgoing front of gas already accreted.

The gas velocity dispersion, measured with respect to the mean cluster velocity, is an indication of both the net gas bulk motion and of small-scale (but still macroscopic) random motions. In general the velocity dispersion decreases towards the cluster center, as shown by the black band in the right plot of Figure 4. However, this global behavior is again the superposition of different populations of particles with contrasting properties, as shown by the colored bands. While following the general trend of decreasing towards the center, the gas accreted at lower and lower redshift has larger and larger net velocities at large radii, and in the case of the most recent accretion $(z<0.2)$ the velocity dispersion is flat at all radii reached by the particles. This is consistent with what we have shown above for the gas thermalization times: the gas accreted more recently is still converting its bulk motions into internal energy. If so, it should also have an average temperature which is lower than the mean at large radii. This is indeed the case, as illustrated in the lower left panel of the left plot of Figure 5. Moreover, the same figure shows that the slower-than-average gas present around the virial radius and accreted at higher redshift is also slightly hotter than the mean, in agreement with the expectation. Finally, the right plots of Figure 5 gives the behavior of gas entropy $S \propto T / \rho^{2 / 3}$, which again confirms this trend, by showing that at large radii the entropy of gas accreted at lower redshift (which is slightly colder and denser) is lower than average, while the opposite is true for gas accreted at higher redshift, which at large radii is lower in density and higher in temperature. 

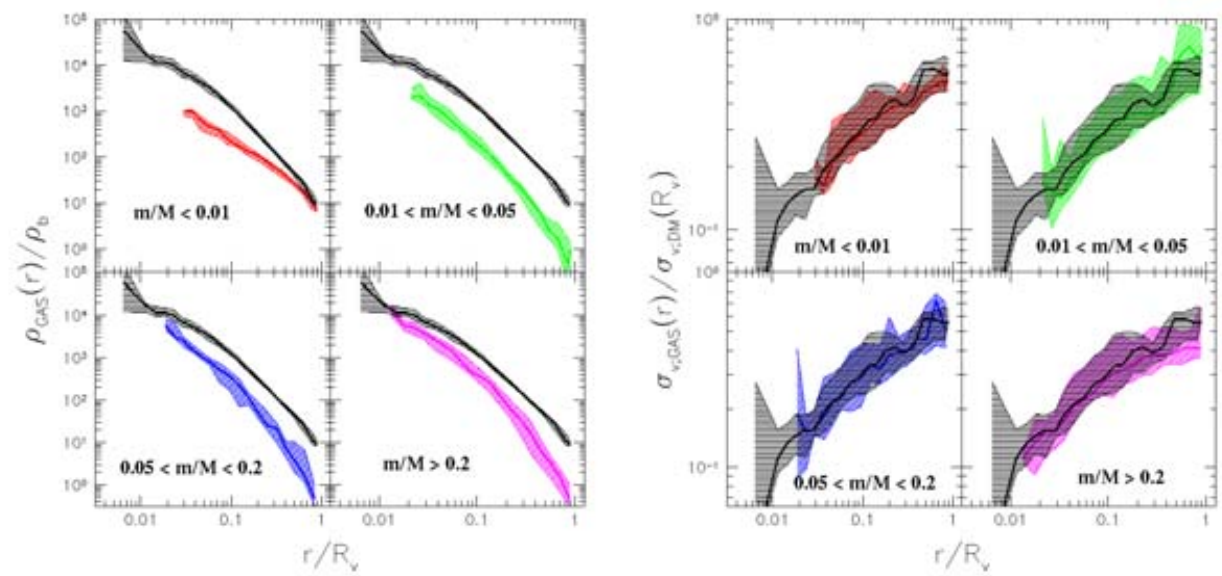

Figure 6. ICM decomposition in satellite mass: gas density (left plot) and gas velocity dispersion (right plot). Colors are as in Figure 4. Here the decomposition is done depending on the mass $m_{v}$ of the satellite carrying the particles into the main cluster of mass $M_{v}$ at merging time. Each panel in each plot refer to a different bin in mass ratio $m_{v} / M_{v}$. For example, the lower right panel in the left plot compares the mean (over the cluster sample) gas density profile made with all particles (black band) with the mean (over the cluster sample) gas profile made only with the particles which accreted in satellites with mass larger than 20 percent of the main progenitor mass, that is, accreted in major mergers.

\subsection{Decomposition in satellite mass}

If we play a similar game of decomposing the gas profiles according to the mass of the satellites that carried the particles, we obtain the profiles of Figures 6 and 7, which are the analogous of Figures 4 and 5 respectively. The gas density profiles in the left plot of Figure 6 clearly show a segregation in gas by this mass selection criterion: gas accreted in small satellites mainly sits in the outer cluster regions, while inner regions are mainly populated by the gas brought in by major mergers. The right plot of the same figure suggests a hint of trend in the outer regions, in the directions of a velocity dispersion lower than the mean both for very small and for very large satellites. In the case of small satellites this could simply reflect the fact that the gas in small satellites is stopped more easily as it enters the main cluster. For major mergers the interpretation is less obvious.

Going to the temperature profiles, in the left plot of Figure 7, we observe a more complex trend. Particles from small satellites end up with a temperature slightly higher then the mean: this is what we would expect if shocks are more efficient in heating the gas up as soon as the satellites enter the main cluster. Satellites with intermediate masses deposit significantly cooler gas in the outer regions: in this case the gas is shielded more efficiently from the stripping and heating. However, going to even larger satellites (lower right panel) we do not observe a continuity in this trend; the resulting temperature profile is well in agreement with the global one. Perhaps this simply reflects the fact that most of the cluster gas is indeed carried in by few massive mergers; in this case the mean cluster temperature would be defined by the temperature of the particles from the most massive satellites.

The right plot in Figure 8 shows the entropy profiles. Now particles from small satellites have density and temperature both higher than the average in the cluster outskirts, therefore the effect on the entropy is almost canceled. Intermediate mass satellites have lower entropy at large radii due to their lower temperature, while massive satellites donate 

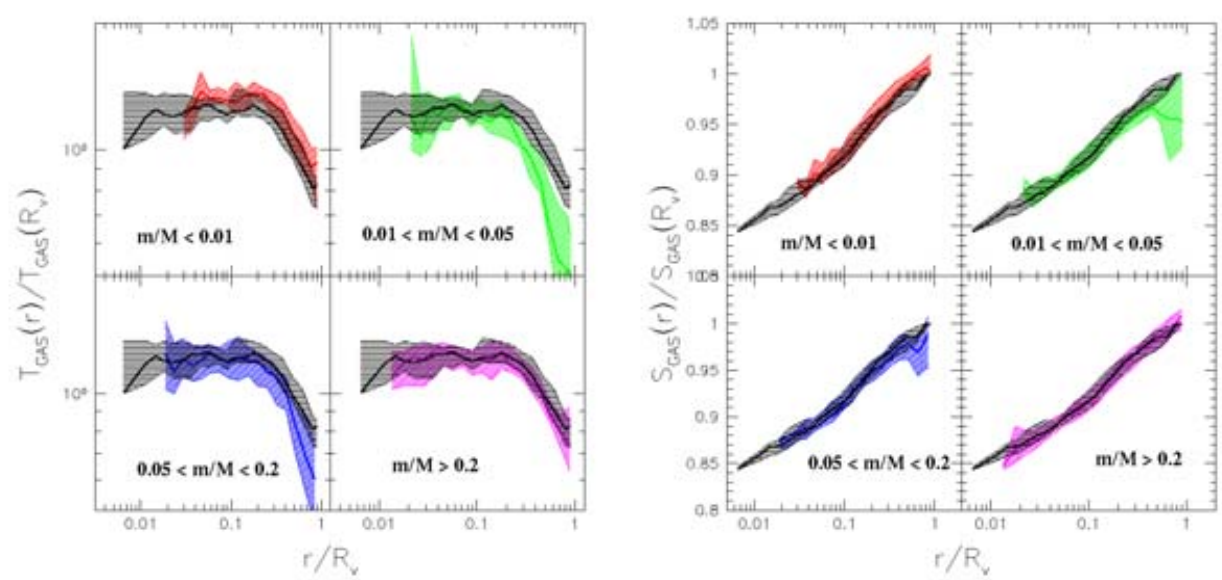

Figure 7. ICM decomposition in satellite mass: gas temperature (left plot) and entropy (right plot). Colors are as in Figure 4.

particles which end up with average entropy. Although it is not easy to understand all these behaviors in detail, we think that this decomposition game can lead to a better understanding of the global properties of clusters observed at any given redshift.

\section{Conclusions}

We have seen that a number of properties of galaxy clusters can be understood by looking at their merging history. The high anisotropy of the matter infall leaves an imprint on the final cluster shape, both in mass and velocity dispersion.

The thermalization of substructure in the main cluster proceeds differently for the outer and inner parts of satellites: while the former are quickly heated to temperatures of the order of the virial temperature of the main cluster, the cores of satellites tend to cool, probably due to the stripping of hot particles (both in dark matter and gas). The decomposition of matter in the final cluster, as a function of either the merging redshift or the satellite mass at merging time, brings further insight into the cluster internal structure, and suggests that at redshift zero different regions of the clusters are unevenly populated by matter accreted in different ways.

\section{Acknowledgements}

I would like to acknowledge Antonaldo Diaferio for organizing so lively a meeting, and for inviting me to give this talk. Partial support was provided by the Italian MIUR (Grant 2001, prot. 2001028932, "Clusters and groups of galaxies: the interplay of dark and baryonic matter") and by ASI. Blackwell Publishing Ltd. is acknowledged for granting the permission to reprint Figures 1,2 and 3 from the original publications.

\section{References}

Tormen, G. 1997 MNRAS 290, 411-421

Tormen, G., Moscardini, L., and Yoshida, N. 2004 MNRAS 350, 1397-1408 